DIE ERSTE SEITE

\section{Erhöhtes kardiovaskuläres Risiko sichtbar gemacht}

\section{Mammografie auch fürs Koronarscreening nutzen?}

Verkalkungen der Brustarterien, die in Screening-Mammografien als Nebenbefund auffallen, korrelieren mit dem koronaren Kalk-Score und sagen das kardiovaskuläre Risiko besser voraus als andere Risikofaktoren. In einer jetzt bei der ACC-Jahrestagung in Chicago vorgestellten Studie erhielten 292 Frauen sowohl eine digitale Mammografie als auch eine Computertomografie des Herzens. In der Mammografie wurden
Kalkablagerungen in den Brustarterien (BAC), im CT Verkalkungen der Koronararterien (CAC) quantifiziert. 70\% der Frauen, die eine BAC aufwiesen, hatten auch eine CAC. Bei jüngeren Frauen betrug die Rate falsch positiver Befunde nur 17\%. Der BAC-Nachweis könnte als Anlass für eine umfangreichere kardiovaskuläre Risikofaktoranalyse dienen, so die Autoren.

- 65. Jahrestagung des American College of Cardiology 2016; J Am Coll Cardiol. 2016. DOI: 10.1016/j.jcmg.2015.10.022

\title{
Lebensverlängernde Stütze
}

\section{Verheiratete haben bessere Chancen bei Krebs}

Bei einer Krebserkrankung ist eine stabile Beziehung von Vorteil: Die Sterblichkeit ist bei Verheirateten deutlich geringer. Epidemiologen vom Cancer Prevention Institute in Fremont analysierten Daten von über 783.000 Tumorpatienten aus dem kalifornischen Krebsregister. Von den Männern waren zum Diagnosezeitpunkt rund 70\% verheiratet, von den Frauen 51\%. Wurden Begleitfaktoren wie Einkommen, Versicherungsstatus und Wohngegend berücksichtigt, lag die Gesamtsterberate bei unverheirateten Männern um 22\%, bei unverheirateten Frauen um 15\% erhöht. Die höchste Mortalität fand sich bei Männern, die noch nie verheiratet waren.

- Cancer 2016; epub 11. April 2016, DOI: 10.1002/cncr.29885.

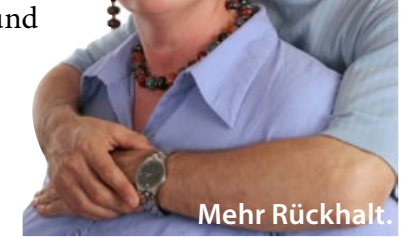

Dr. med. Dirk Einecke Chefredakteur dirk.einecke@springer.com

Früher Schlaganfall

\section{Für Frauen besonders folgenschwer}

Viele Patienten, die als junge Erwachsene einen Schlaganfall erlitten haben, bleiben langfristig auf Hilfe angewiesen. Die Aussichten für Frauen sind besonders schlecht.

Niederländische Neurologen haben 619 Patienten, die im Alter zwischen 18 und 50 Jahren wegen eines Schlaganfalls oder einer TIA behandelt worden waren, nachbeobachtet. Dazu wurden der funktionelle Status (modifizierte Rankin-Skala, mRS) und die Alltagskompetenz (mit dem instrumentellen Activities of Daily Living, iADL) erfasst. Im Lauf von 14 Jahren hatten 90 Patienten $(14,5 \%)$ einen weiteren Schlaganfall erlitten. Von den 423 Patienten mit initialem Schlaganfall hatten 189 $(44,7 \%)$ und von den 196 TIA-Patienten 48 $(24,5 \%)$ einen ungünstigen mRS-Wert $>2$, wobei die betroffenen Frauen jünger und die zerebralen Ischämien bei ihnen weniger gravierend gewesen waren. Ein schlechtes Ergebnis im iADL hatten $57(22,9 \%)$ Insult- und $34(15,2 \%)$ TIA-Patienten. Als Hauptrisikofaktoren für eine ungünstige Langzeitprognose erwiesen sich weibliches Geschlecht $(\mathrm{OR}=2,0)$ und der Schweregrad des Schlaganfalls (OR $=1,2)$.

- J Neurol 2016; epub 2.4.16, DOI: 10.1007/s00415-016-8042-2.

\section{Populationsbasierte Kohortenstudie \\ Epilepsie-Risiko für Typ-1-Diabetiker dreifach erhöht}

Kinder mit Typ-1-Diabetes haben einer taiwanesischen Kohortenstudie zufolge ein stark erhöhtes Risiko, eine Epilepsie zu entwickeln. In die retrospektive Analyse wurden 2.568 Typ-1-Diabetiker (mittleres Alter 10,4 Jahre) eingeschlossen und mit jeweils zehn gesunden Kindern verglichen. Nach Berücksichtigung verschiedener Faktoren wie Alter, Geschlecht, Komorbi- ditäten und geringes Geburtsgewicht ergab sich für Kinder mit Typ-1-Diabetes ein fast dreimal so hohes Epilepsierisiko wie in der Kontrollgruppe (Epilepsie-Inzidenz 33,7 vs. 10,4/10.000 Personenjahre; HR $2,84)$. Die Autoren führen dies u. a. auf ZNS-Schäden infolge von Hyper- und Hypoglykämien zurück.

- Diabetologia, online 31. März 2016
SPRINGERMEDIZIN.DE AUF FACEBOOK

Hausärzte und Internisten können springermedizin.de jetzt auch auf Facebook kontaktieren:

$\rightarrow$ www.facebook.com/SpringerMedizin.Hausarzt

$\rightarrow$ www.facebook.com/SpringerMedizin.Innere 